Check for updates

Cite this: RSC Adv., 2017, 7, 27496

\title{
Proteomic study of the effect of different cooking methods on protein oxidation in fish fillets
}

\author{
Lyulin $\mathrm{Hu}{ }^{a}$ Sijie Ren, ${ }^{a}$ Qing Shen, ${ }^{a}$ Jianchu Chen, (D) *abc Xingqian Ye ${ }^{\text {abc }}$
} and Jiangang Ling ${ }^{d}$

The effect of different cooking methods on protein and lipid oxidation of farmed sturgeon (Acipenser gueldenstaedtii) was investigated. In this study, the fish fillets were cooked using five different methods (boiling, steaming, microwaving, roasting and deep-frying), to an internal temperature of approximately $85{ }^{\circ} \mathrm{C}$. General indexes for protein oxidation (carbonyls, free thiols and Schiff bases) and lipid oxidation were evaluated by spectrophotometric and fluorimetric methods. Side-chain modifications of amino acid residues were pinpointed by gel- and MS-based proteomic technology. The results are as follows: (1) cooking, especially roasting and frying, significantly increased the levels of carbonyls and Schiff bases and decreased the amounts of free thiols. (2) Oxidation of aromatic amino acids and extensive oxidation of lysine to carbonyl-containing compounds were characterized in all the cooked samples. (3) Indirect protein oxidation caused by interacting with lipid-derived aldehydes like malondialdehyde (MDA) and 4hydroxynonenal (HNE) was also observed and much more pronounced in roasted and fried samples, giving a footprint of lipid-protein interaction during cooking. In summary, cooking treatment can induce protein oxidation in sturgeon fillets with a variety of modifications occurring at the protein primary structure. Molecular mapping of protein oxidation is conducive to understand the mechanism of cooking-induced protein oxidation and allows better control of the nutritional properties of protein-

based products.

Received 23rd March 2017 Accepted 15th May 2017

DOI: $10.1039 / c 7 r a 03408 c$

rsc.li/rsc-advances

\section{Introduction}

Fish has long been recognized as a great dietary source of highquality proteins and polyunsaturated fatty acids (DHA, EPA, etc.). Though sometimes eaten raw (e.g., sashimi), fish is nearly always cooked prior to its consumption for the sake of inactivation of pathogens and enhancement of palatability. Cooking procedures including boiling, steaming, frying, roasting, will induce a cascade of physicochemical reactions among which lipid oxidation is one of the most pronounced because the secondary products are volatile compounds that can be readily detected by our body's olfactory cells - rancidity, off-flavor, and, sometimes on the positive aspect, aroma. ${ }^{1}$ Much literature is available on the investigation of the effect of cooking on lipid oxidation of meat and fish, ${ }^{2-4}$ while protein oxidation, the "poor cousin" of lipid oxidation portrayed by Estévez et al. ${ }^{5}$ has been always neglected over the past decades due to its less perceivable influence exerted on meat, more complicated oxidative mechanisms and greater difficulty in detection.

${ }^{a}$ College of Biosystems Engineering and Food Science, Zhejiang University, Hangzhou 310058, China. E-mail: jc@zju.edu.cn

${ }^{b}$ Fuli Institute of Food Science, Zhejiang University, Hangzhou 310058, China

'Zhejiang Key Laboratory for Agro-Food Processing, Zhejiang R\&D Center for Food Technology and Equipment, Hangzhou 310058, China

${ }^{d}$ Ningbo Academy of Agricultural Sciences, Ningbo, Zhejiang 315040, China
Heat treatment has been proved to induce protein oxidation which leads to a variety of changes at molecular level, involving gain of carbonyl derivatives, reduction of thiol groups, loss of tryptophan fluorescence, accumulation of Schiff-base structures (SB) and formation of intra and intermolecular cross-links, ${ }^{5}$ which could subsequently affect physicochemical and organoleptic characteristics of meat, including texture deterioration induced by loss of water holding capacity, ${ }^{6}$ and alteration of flavor through interaction between secondary lipid oxidation products and protein.

Dietary protein oxidation, similar to in vivo protein oxidation-a source of aging and diseases, might have a potential impact on human health, ${ }^{6}$ through reducing the amount of essential amino acids, impairing the protein digestibility and generating harmful compounds like $\alpha$-aminoadipic semialdehyde (AAS), kynurenines, etc.

In view of the abovementioned facts, it is crucial to figure out the occurrence, intensity, and consequences of the protein oxidation during cooking. Many relevant studies have been carried out in this field. Traore, et al. ${ }^{7}$ reported $45 \%$ and $83 \%$ increase in carbonyl groups when pig meat was boiled for 10 and $30 \mathrm{~min}$ respectively. Hernández-López, et al. ${ }^{8}$ detected about $50 \%$ loss in thiol content with a marked increase in disulfide bonds in cooked pork. An increase in the amount of AAS in the protein after cooking was also reported. ${ }^{9}$ Gatellier, 
et $a .^{10-12}$ have done a great deal of related work, apart from demonstrating aromatic amino acid residues were seriously damaged when heated at 100 and $140{ }^{\circ} \mathrm{C}$, these authors also studied the oxidation in meat heated at varied temperatures and for different periods of times and found the correlations between protein carbonyls, lipid oxidation, and SB formation. Moreover, Sun, et al. ${ }^{13}$ showed that high temperature intensified the protein-bound $N \varepsilon$-carboxymethyllysine (CML) and $N \varepsilon$-carboxyethyllysine (CEL) - two advanced glycation end products (AGEs, formed via the Maillard reaction). However, on one hand, these papers merely targeted time-temperature combinations of one type of cooking method, not the comparison between different cooking techniques. On the other, red meats, including pig, beef and lamb, not the fish, were the major materials for their experiments. The scarcity of studies on protein oxidation of fish treated with various cooking methods prompts us to probe into this field.

A holistic approach is needed to evaluate protein oxidation due to its complex oxidative mechanisms and a wide range of oxidation products. Over the past few years, MS-based proteomics has emerged as an innovative technique, and has been successfully applied by many research teams to pinpoint the protein primary-level modifications of food (especially dairy and meat) exposed to oxidative and other insults. Using a redox proteomics approach, Dyer, et al. ${ }^{\mathbf{1 4 , 1 5}}$ characterized residue sidechain modifications and tracked their key sites within the milk proteins, e.g., lactoferrin and $\beta$-lactoglobulin which were exposed to heating or ultraviolet $\mathrm{B}$ radiation. In addition, DebChoudhury, et al. ${ }^{16}$ observed increasing oxidative modifications of beef boiled in water with increased heating time. Similarly, LC-MS-MS results from Yu, et al. ${ }^{17,18}$ revealed various oxidative modifications of aromatic residues in boiled and roasted lamb. In conclusion, MS-based proteomics help detect low abundance changes at the molecular level, which provides insight into the mechanism of cooking-induced protein oxidation and allows effective control of nutritional values of meat and fish products.

Sturgeons are important commercial fish species which are appreciated not only because of their highly valuable caviar, but also for their boneless flesh with excellent quality and taste. The effect of different cooking methods on fatty acids composition and lipid oxidation of sturgeon meat have been studied. ${ }^{19,20}$ But there is no literature available on protein oxidation of sturgeon induced by different cooking methods. Hence, in this paper, we aim to evaluate the effect of five cooking methods (boiling, steaming, microwaving, roasting and frying) on some general indices of protein oxidation and modifications in amino acid residues using sodium dodecyl sulfate-polyacrylamide gel electrophoresis (SDS-PAGE) in conjunction with ultra performance liquid chromatography-mass spectrometry/mass spectrometry (UPLC-MS/MS).

\section{Materials and methods}

\section{Chemicals}

$4-20 \%$ T precast gel $(100 \times 80 \times 1 \mathrm{~mm})$, TruPAGE LDS sample buffer, dithiothreitol (DTT), wide-range protein marker, and iodoacetamide (IAA) were purchased from Sigma-Aldrich Co. (St. Louis, MO, USA). Sequencing grade trypsin was purchased from Promega. Co. (Madison, WI, USA). 2,4-Dinitrophenylhydrazine (DNPH), thiobarbituric acid and 2,2'-dithiobis (5nitropyridine) DTNP were purchased from Aladdin Bio-Chem Technology Co. Ltd. (Shanghai, China). Modified braford protein assay kit and Coomassie Brilliant Blue R-250 were purchased from Sangon Biotech Co., Ltd. (Shanghai, China). All the other chemical reagents, including ethanol, $\mathrm{HCl}$, etc., used in the experiments were analytical grade and purchased from Sinopharm Chemical Reagent Co., Ltd. (Shanghai, China).

\section{Samples}

Decapitated and eviscerated frozen sturgeon (Acipenser gueldenstaedtii) was purchased from Qiandaohu Xunlong Sci-tech Co. Ltd., (Hangzhou, China) and was transported in cold iced boxes to laboratory within one hour.

\section{Samples preparation and cooking methods}

Frozen fish was thawed in refrigerator at $4{ }^{\circ} \mathrm{C}$ overnight. Thawed samples were chunked and cut into pieces $(8 \pm 0.5 \mathrm{~cm}$ in side length, $1.5 \pm 0.2 \mathrm{~cm}$ thick), then they were divided into 6 groups. The first group was the control (uncooked); the other 5 groups were cooked by five different methods (boiling, steaming, microwaving, oven cooking and frying) to an internal temperature of $85 \pm 5{ }^{\circ} \mathrm{C}$. No extra ingredients were added to avoid interference.

Boiling treatment. The fish fillets were put into a pot (Supor, China) filled with boiling water (ratio of fish to water is $1: 6(\mathrm{w} /$ v)) and cooked for $8 \mathrm{~min}$ with pot covered.

Steaming treatment. The fillets were uniformly placed on a stainless steel tray and put into a steamer (Fotile, SCD20-01, China) and cooked with $100{ }^{\circ} \mathrm{C}$ water vapor for $8 \mathrm{~min}$.

Microwaving-cooking. The fillets were placed into a microwaveable bowl and cooked in a microwave oven (Fotile, W2580001AG, China). Experiment was performed at $800 \mathrm{~W}$ for $6 \mathrm{~min}$ with each side for $3 \mathrm{~min}$.

Roasting. The fillets were regularly placed on a baking tray and heated in convection oven set to $200{ }^{\circ} \mathrm{C}$. During cooking, samples were heated for $10 \mathrm{~min}$ on each side.

Frying. The fillets were uniformly placed in a wire mesh basket and immersed in $180^{\circ} \mathrm{C}$ soybean oil in a fryer (Vesta EF81, China) for $5 \mathrm{~min}$.

The five different cooking methods were performed in triplicate. After heat treatments, surface moisture and grease were removed from samples, and all the samples were cooled to room temperature. Each sample of raw or cooked fish fillets was crushed using a kitchen blender (Sun, China). The ground samples were packaged in PVDC bag and stored at $-80{ }^{\circ} \mathrm{C}$ until use.

\section{Determination of thiobarbituric acid-reactive substances}

TBARS values were assessed by a method described by Ganhão, et al. ${ }^{21}$ using thiobarbituric acid. The results were expressed as mean mg malondialdehyde (MDA) per $\mathrm{kg}$ meat \pm SD. All measurements were performed in triplicate. 


\section{Measurement of carbonyl groups}

The levels of protein carbonyl groups were measured by the method described by Mercier, et al. ${ }^{22}$ Carbonyl groups were detected by reactivity with DNPH to form protein hydrazones. The results were expressed as mean nanomoles of DNPH fixed per milligram of protein. All measurements were performed in triplicate.

\section{Measurement of free thiols}

The concentration of thiol oxidation was quantified by a modification of Ellman's method using DTNP. ${ }^{23}$ The results were expressed as mean nmol thiol per $\mathrm{mg}$ protein \pm SD. All measurements were performed in triplicate.

\section{Fluorescence measurements of Schiff base structures (SB)}

The fluorescence emission of Schiff base structures (SB) were assessed by the method of Gatellier, et al. ${ }^{\mathbf{1 0}}$ using an extraction in dichloromethane/ethanol mixture and the fluorescence intensity (FI) of the upper phase were measured with a Cary Eclipse fluorometer (Agilent Technologies, Santa Clara, CA, USA). Emission spectra of SB were recorded from 380 to $600 \mathrm{~nm}$ with the excitation wavelength set at $360 \mathrm{~nm}$. Excitation and emission slit were set at $5 \mathrm{~nm}$. All measurements were performed in duplicate, and FI values were expressed in arbitrary units.

\section{SDS-PAGE gel electrophoresis}

Samples were weighed and soaked in urea-thiourea solution [7 M urea, $2 \mathrm{M}$ thiourea, and 2\% freshly added dithiothreitol (DTT)] at $1: 15$ ratio $(\mathrm{w} / \mathrm{v})$. Then the slurries were homogenized and centrifuged to obtain the supernatants of which protein concentration was determined by modified braford protein assay kit. Subsequently, the urea-thiourea extract was mixed with TruPAGE LDS sample buffer in $3: 1$ ratio (v/v) and heated at $70{ }^{\circ} \mathrm{C}$ water bath for $10 \mathrm{~min}$. About $20 \mu \mathrm{g}$ of protein was run on a $4-20 \% \mathrm{~T}$ precast gel in a VE-180 Mini Verticel Gel Electrophoresis Cell (Tanon Science \& Technology Co., Ltd. Shanghai, China) at a constant voltage of $150 \mathrm{~V}$ for $70 \mathrm{~min}$. Then the gel was stained for $1.5 \mathrm{~h}$ in Coomassie Brilliant Blue R-250, followed by being decolorized in a solution containing $45 \%$ methanol, $10 \%$ acetic acid and $45 \%$ water. Each gel was scanned using a 2500R gel imaging system (Tanon Science \& Technology Co., Ltd. Shanghai, China).

\section{In-gel trypsin digestion}

Gel bands at around $42 \mathrm{kDa}$ of all the samples were cut out $(5 \times$ $2 \mathrm{~mm}$ ) and washed in $100 \mathrm{mM}$ ammonium bicarbonate/30\% acetonitrile at $37{ }^{\circ} \mathrm{C}$ with gentle shaking for $30 \mathrm{~min}$ and with one change of the destaining solution. Samples were reduced at $37{ }^{\circ} \mathrm{C}$ under gentle shaking $(500 \mathrm{rpm})$ for $45 \mathrm{~min}$, washed twice with $100 \mathrm{mM}$ ammonium bicarbonate and then alkylated with $100 \mu \mathrm{L}$ of $0.05 \mathrm{M}$ IAA. After twice washing with $50 \mathrm{mM}$ ammonium bicarbonate, each sample was incubated in 100\% acetonitrile at $15{ }^{\circ} \mathrm{C}$ for $10 \mathrm{~min}$. Acetonitrile was then removed and the samples were dried in a vacuum centrifuge (Labconco,
Kansas City, MI, USA). Each dried sample was digested with 0.5 $\mu \mathrm{g}$ of sequencing grade trypsin in $40 \mu \mathrm{L}$ of $\mathrm{NH}_{4} \mathrm{HCO}_{3}$ buffer at $37{ }^{\circ} \mathrm{C}$ overnight. Tryptic digests were extracted sequentially with $10 \%$ acetonitrile in $50 \mathrm{mM}$ ammonium bicarbonate, $50 \%$ acetonitrile/1\% formic acid and $80 \%$ acetonitrile. Pooled extracts for each sample were dried in the vacuum centrifuge at $30{ }^{\circ} \mathrm{C}$ and stored at $-80{ }^{\circ} \mathrm{C}$ until analysis.

\section{UPLC/MS/MS methods and data analyses}

The tryptic digest of each slice was resuspended in $50 \mu \mathrm{L}$ of $2 \%$ acetonitrile/ $0.5 \%$ formic acid. The peptide mixtures were injected onto the trap column with a flow rate of $10 \mu \mathrm{L} \mathrm{min}{ }^{-1}$ for 2 min using a Thermo Scientific Easy nanoLC 1000 (Waltham, MA, USA). The trap was equilibrated as a maximum pressure of 500 bar for $12 \mu \mathrm{L}$ followed by column equilibration at a maximum of 500 bar for $3 \mu \mathrm{L}$ before starting gradient elution of column. The samples were subsequently eluted with a fivestep linear gradient (A: $\mathrm{ddH}_{2} \mathrm{O}$ with $0.1 \%$ formic acid, B: ACN with $0.1 \%$ formic acid): $0-10 \mathrm{~min}, 3-8 \% \mathrm{~B} ; 10-120 \mathrm{~min}, 8-20 \%$ B; 120-137 $\mathrm{min}, 20-30 \% \mathrm{~B}$; $137-143 \mathrm{~min}, 30-90 \% \mathrm{~B}$; $143-$ $150 \mathrm{~min}, 90 \% \mathrm{~B}$. The column flow was maintained as $250 \mathrm{~nL}$ $\min ^{-1}$. The chromatographic system was composed of a trapping column $(75 \mu \mathrm{m} \times 2 \mathrm{~cm}$, nanoviper, C18, $3 \mu \mathrm{M}, 100 \AA)$ and an analytical column $(50 \mu \mathrm{m} \times 15 \mathrm{~cm}$, nanoviper, C18, $2 \mu \mathrm{M}$, $100 \AA$ A). Data collection was performed using Thermo LTQOrbitrap Velos Pro equipped Nanospray Flex ionization source and FTMS (Fourier transform ion cyclotron resonance mass analyzer) analyzer combined with Thermo LTQ-Orbitrap Elite equipped with Ion Trap analyzer. The parameters for FTMS were as follows: data collection were at $60 \mathrm{~K}$ for the full scan MS, positive as polarity, profile as data type, and then proceeded to isolate the top 20 ions for MS/MS by CID $(1.0 \mathrm{~m} / \mathrm{z}$ isolation width, 35\% collision energy, 0.25 activation Q, $10 \mathrm{~ms}$ activation time). Scan range was set as $300 \mathrm{~m} / z$ first mass and $2000 \mathrm{~m} / \mathrm{z}$ last mass. The parameters for Ion Trap analyzer were normal mass range, rapid scan rate, and centroid data type. Independent duplicates were used for mass spectrometric analysis.

SEQUEST HT search engine configured with Proteome Discoverer 1.4 workflow (Thermo Fischer Scientific, Bremen, Germany) was used for mass spectrometer data analyses. Fish peptides database from Uniprot were configured with SEQUEST HT for searching the datasets. The search parameters include $10 \mathrm{ppm}$ and $0.8 \mathrm{Da}$ mass tolerances for MS and MS/MS respectively, trypsin as the proteolytic enzyme with two allowed missed cleavage, oxidation and deamidated as dynamic modifications, and carbamidomethyl as static modification. Besides, additional variable modifications (Table 1), known as potential heating and ROS induced ones, divided into 4 groups (each group for one search) were included. Furthermore, peptides were extracted using high peptide confidence. 1\% FDR (false discovery rate) was calculated using a decoy database by searching the peptide sequence.

\section{Statistics}

Data were expressed as the mean \pm SEM (standard error of mean). The effect of the different cooking methods on carbonyl 
Table 1 List of heating and/or reactive oxygen species-induced modifications

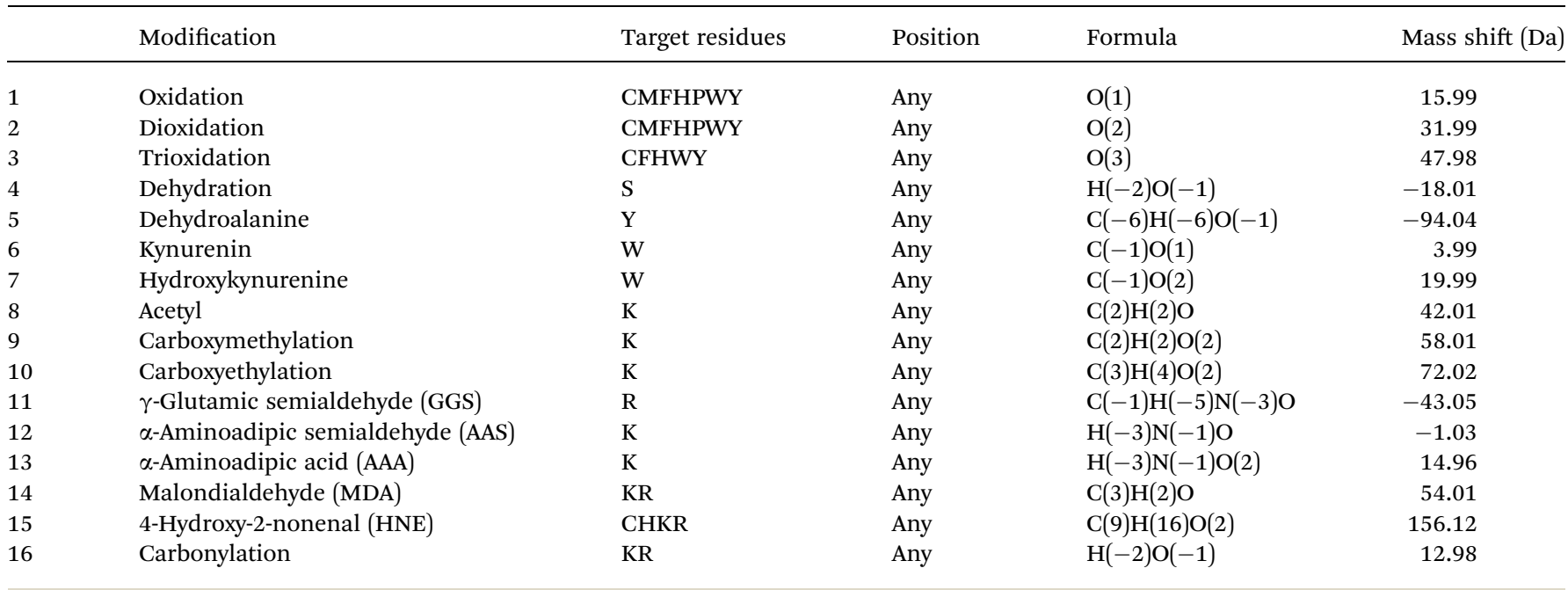

groups, free thiols and TBARS was analyzed by one-way analyses of variance (ANOVA) using SPSS for Mac (v. 23.0). Differences were considered to be significant at $p<0.05$. Tukey's tests were performed when significant overall differences were found.

\section{Results and discussion}

\section{Oxidation in cooked fish fillets with different cooking methods}

This part is aimed to provide a broad view of lipid and protein oxidation in different types of cooked fish fillets using several common biochemical measurements. After cooking, oxidative changes of the fish fillets were detected (Table 2). Our results showed that the extent of oxidation were in general more remarked in roasted and fried fillets, while boiling and steaming, two relatively mild cooking methods with a maximum temperature at $100{ }^{\circ} \mathrm{C}$, rendered less oxidation in lipid and protein and the effect of microwaving cooking was in between, indicating that the types of cooking really matter in lipid and protein oxidation development. Oxidation in meat induced by cooking can be partly attributed to the fact that the meat loses its natural antioxidant properties when heated. What's more important is that heat can post a damage to cellular structure of meat, making it more exposed to oxygen, then triggers the reactive oxygen species (ROS) which subsequently attack lipid and protein molecules. In addition, cooking contributes to the oxidative cleavage of the porphyrin ring, thus heme iron, one of the pro-oxidants, will be released to accelerate the oxidative deterioration..$^{24}$

Lipid oxidation. Lipid oxidation, a free radical chain reaction, gives rise to some primary products such as conjugated dienes and hydroperoxides, and these compounds will readily decompose to form ketones, alcohols, and aldehydes, which are considered secondary products of lipid oxidation. ${ }^{25}$ Since there is an intimate link between lipid and protein oxidation, lipid oxidation can be an important indicator to study protein oxidation.

The extent of lipid oxidation is expressed by the level of TBARS which represents the content of secondary oxidation products - mainly aldehydes like MDA. TBARS production in fish fillets varied with different ways of cooking (Table 2). Cooking promoted the oxidative changes of lipids which was reflected in significantly higher TBARS values in the cooked samples (from $0.61 \mathrm{mg}$ MDA equivalents per $\mathrm{kg}$ in raw material to $0.73,0.72,0.92$ and $0.77 \mathrm{mg}$ MDA equivalents per $\mathrm{kg}$ after boiling, steaming, microwaving and frying, respectively). TBARS accumulation has been widely reported in variant types of cooked meat and fish as compared to the raw materials. However, we were surprised to find that the roasted samples contained a significantly lower level of TBARS (0.52 mg MDA

Table 2 The content of carbonyl groups, free thiols and TBARS value in sturgeon fillets after cooking (boiling, steaming, microwaving, roasting and frying) ${ }^{a}$

\begin{tabular}{|c|c|c|c|c|c|c|}
\hline Oxidation indexes & Control & Boiling & Steaming & Microwaving & Roasting & Frying \\
\hline Free thiols $\left(10^{2}\right.$ nmol per mg protein) & $0.80 \pm 0.02^{\mathrm{a}}$ & $0.53 \pm 0.02^{\mathrm{b}}$ & $0.54 \pm 0.03^{\mathrm{b}}$ & $0.45 \pm 0.02^{\mathrm{c}}$ & $0.30 \pm 0.01^{\mathrm{e}}$ & $0.37 \pm 0.01^{\mathrm{d}}$ \\
\hline TBARS (mg MDA per kg meat) & $0.61 \pm 0.01^{\mathrm{e}}$ & $0.73 \pm 0.01^{\mathrm{b}}$ & $0.72 \pm 0.01^{\mathrm{b}}$ & $0.92 \pm 0.02^{\mathrm{a}}$ & $0.52 \pm 0.01^{\mathrm{d}}$ & $0.77 \pm 0.02^{\mathrm{b}}$ \\
\hline
\end{tabular}

${ }^{a}$ a-e Means $(n=3)$ with different superscripts within the same row were significantly different $(p<0.05)$. 
equivalents per $\mathrm{kg}$ ) which was supposed to be higher compared with the control. In fact, there were still several authors who found similar results with us. Utrera, et al. ${ }^{\mathbf{2 6}}$ reported a decrease of TBARS in beef patties compared with the raw after cooking at $170{ }^{\circ} \mathrm{C}$ for $18 \mathrm{~min}$. A lower level of TBARS in beef meat cooked at a higher temperature and for a longer time was described by Roldan, et al. ${ }^{9}$

A probable explanation to the decreased TBARS value could be that, besides decomposing aldehydes - secondary lipid oxidation products - to volatile compounds, lengthy exposure to more intense cooking condition apparently favors further reactions between these aldehydes and other meat components, such as proteins. ${ }^{27}$

Though TBARS value in fried fish fillets was much higher than the raw samples, it was not statistically significant compared with the boiled and steamed samples. Lipid oxidation might be more complicated during frying than other cooking methods. On one hand, the TBARS can be produced from both fish oil and frying oil, thus the TBARS value was supposed to be higher in fried samples which sucked up much frying oil, on the other, similar to the roasting condition, high frying temperature could not only induce TBARS but prompted them further to react with other components, resulting in the reduction of TBARS as well. Due to the trade-off of abovementioned processes, the effect of the frying on the development of lipid oxidation was not significantly higher than the other cooking methods.

Carbonyl groups. The level of protein carbonyls, which is widely acknowledged as an indicator of protein oxidation, was determined by the DNPH method. Carbonyl content of the fish samples cooked with different methods and the control was shown in Table 2. Increased carbonyls were observed in all the cooked samples and more obviously, roasted and fried fish fillets exhibited a massive increase of carbonyl content (around 4 -fold increase compared to the raw) than the samples cooked by boiling, steaming and microwaving (94\%, 91\% and 208\% increase compared with the raw, respectively). All these results were of the same order with those described in other studies. ${ }^{7,11}$

Different from other cooking methods, frying enhanced the oil absorption in the fillets, which made the protein more exposed to the oxidizing lipids. Since oxidizing lipid is believed to be an impeller of protein oxidation, ${ }^{28}$ apart from direct oxidation of side-chain amino acids (arginine, histidine, lysine, etc.) attacked by ROS induced by heating, the transfer of lipidderived radicals from oxidizing lipid to protein, ${ }^{29}$ or covalent binding of lipid-derived aldehydes to protein could also explain the much higher level of carbonyls in the fried fish fillets. Although, no extra oil was added to the roasted samples, highest carbonyl content observed could be attributed to the longer heating time with a higher temperature which were key factors contributing to protein and lipid oxidation, or even the reaction between them.

Free thiols. Thiol groups from cysteine residues are particularly vulnerable to attacks by ROS and other radicals, undergoing a wide array of oxidative changes. Thus the level of free thiols calculated by DNTS method serves as another indicator for protein oxidation.
In the present study, a significant loss of free thiols was observed in the fillets cooked with different methods (Table 2), and thiol oxidation was relatively intensive in roasted (62\% loss compared to the raw) and fried (54\% loss) samples compared to boiled (34\% loss), steamed (32\% loss) and microwaved (43\% loss) ones.

Basically, loss of free thiols could be ascribed to two major pathways: reaction with other thiol/thiolate to form disulfide bonds (a reversible oxidation) and reaction with $\mathrm{O}_{2}$ to form other thiol oxidation products, such as sulfenic acid (CysSOH), sulfinic acid $\left(\mathrm{CySSO}_{2} \mathrm{H}\right)$, and sulfonic acid $\left(\mathrm{CySSO}_{3} \mathrm{H}\right){ }^{30}$

Schiff bases (SB). Schiff base (SB), a type of organic compounds containing $\mathrm{C}-\mathrm{N}$ double bond, can be generated by the reaction between the free amino groups (lysine, arginine, and glutamine, etc.) of proteins and aldehydes from lipid peroxidation or reducing sugars. ${ }^{31}$ Since these species can emit fluorescent light, the fluorescence spectrometer was used to evaluate the content of SB.

Formation of SB in the cooked fish fillets and the control were demonstrated in Fig. 1 which displayed varied patterns of fluorescence emission spectra of different samples. The boiling and steaming treatments merely had a slight impact on the fluorescence intensity, while the roasting and frying exerted a notable effect on the spectra, with a maximum increasing by around 10 times than that of the control group. The huge gap observed in peak values between those samples revealed that the production of fluorescent pigments was dependent on high temperature.

In raw meat and the samples under the less intense cooking conditions (boiling, steaming and microwaving), the patterns of spectra were more complex with two peaks (the first peak observed at around $405 \mathrm{~nm}$ and the second at $515 \mathrm{~nm}$ ). However, only one peak, shifting from $405 \mathrm{~nm}$ to $425 \mathrm{~nm}$, was observed under the extreme cooking methods (roasting and frying). An overlap with the first peak could be the major reason for the vanishing of the second peak of roasted and fried samples.

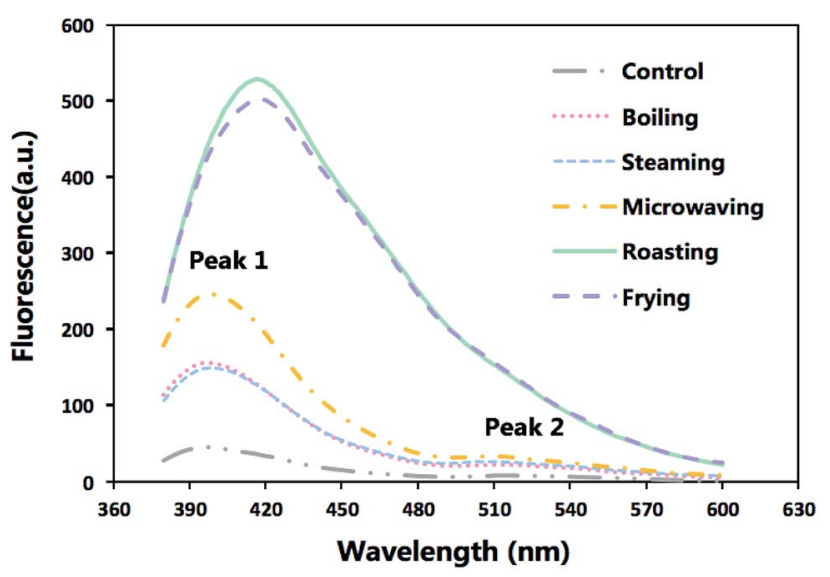

Fig. 1 The emission fluorescence spectra of extracts from raw sturgeon fillets and those cooked with different methods after excitation at $360 \mathrm{~nm}$. 
According to Gatellier, et al., ${ }^{\mathbf{1 0}}$ higher SB formation in samples after roasting and frying treatment can be attributed to probable interactions between proteins and lipid oxidation products (aldehydes).

Besides, the visible shift of maximum emission wavelength (Fig. 1) suggested that different heating process would give rise to the production of SB with different chemical structures but the jury is still out on the precise chemical structure of these compounds.

Gel-based and MS-based proteomic study on modifications of amino acids

SDS-PAGE gels. Though in our study, gel electrophoresis was used to preliminarily separate the fish proteins for further analysis, it helps a lot to assess protein aggregation, degradation, or loss in solubility upon cooking.

The SDS-PAGE profiles demonstrated some alterations in banding patterns between the protein samples treated with 5 cooking methods and the control (Fig. 2), which revealed that different proteins exhibited variant degrees of tolerance to heating. The control and the boiled, steamed and microwaved samples showed relatively distinct but somewhat different banding patterns, while the roasted and fried sample exhibited less defined binds above around $50 \mathrm{kDa}$. The most remarkable changes among all these bands were loss of myosin heavy chain (MHC) and upshift of the band. The MHC was highly abundant in the control, boiled and steamed samples according to the band intensity which moderately weakened in the samples treated by microwaving and almost diminished under roasting and frying. Not surprisingly, samples subjected to the harshest treatment had the lowest content of MHC as was consistent with MHC being distinctively intolerant to heating reported by many authors. ${ }^{32,33}$

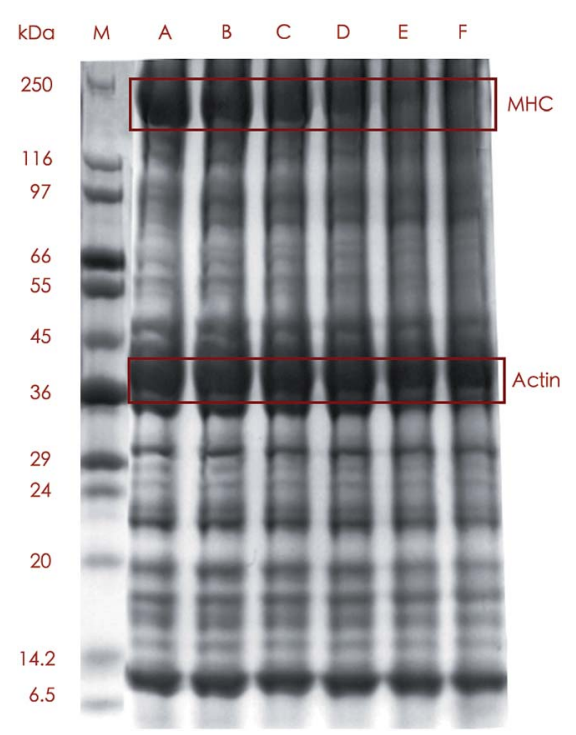

Fig. 2 Representative SDS-PAGE gel lanes of protein extracts from raw sturgeon fillets and those cooked with different methods $(M$ : marker; $A$ : raw sample; $B$ : boiled sample; $C$ : steamed sample; $D$ : microwaved sample; E: roasted sample; F: fried sample; MHC: heavy myosin chain).
The aforementioned results could be explained both by formation of polymerized protein aggregates of which some were too large to get into the gels, ${ }^{33}$ and by fragmentation of MHC into smaller protein molecules which, in our study, was confirmed by the detection of MHC-derived peptides in the digest of actin from cooked samples (data not shown).

Protein aggregation, one of the major protein structural changes, has important implications for the food, since it could affect protein digestibility. Cooking can induce protein aggregation which involves both non-covalent and covalent binding. We can not exclude the possibility that thermal denaturation processes, leading to an increase in protein surface hydrophobicity, serves as a vital role in cooking-induced protein aggregation. Several types of cross-links involved in oxidative reactions have also been shown to be relevant in aggregation of meat protein upon cooking, including dityrosine, Schiff bases, disulfide bridges, and protein carbonylation. ${ }^{17}$

In great contrast with $\mathrm{MHC}$, actin, another one of the most abundant proteins in fish, was much more stable when subject to heating since almost no visible alterations were shown in the bands from cooked meat even after extreme cooking condition like roasting, which was in line with some other studies. Actin was much more stable and a decrease in the actin band did not occur until $20 \mathrm{~min}$ of heating at $140{ }^{\circ} \mathrm{C} .{ }^{33} \mathrm{Huang}$, et al. ${ }^{34}$ found actin bands were much the same at any temperatures in their experiment.

Modifications of amino acid residues. Almost all amino acids residues are sensitive to oxidative stress, resulting in various types of modifications. Both the abundance and thermal stability render the actin an ideal target for mapping cooking-induced modifications which was also supported by $\mathrm{Yu}$, et al. ${ }^{17}$ who identified that actin was a major source of observed heat-induced modifications.

Sequence coverage can be a broad indicator of protein modification, for increasing cross-link formation (protein backbone cleavage) tends to result in decreased (increased) sequence coverage. ${ }^{\mathbf{1 4}}$ Interestingly, we observed decreased sequence coverage in the actin from boiled and roasted samples but increased sequence coverage in the roasted and fried samples (data not shown). An explanation for these could be that relatively mild heating conditions (like boiling and steaming) trigger more aggregation in protein, while harsh conditions (like roasting and frying) favor a tendency for fragmentation.

Heat-induced side-chain modifications observed in actin from different types of cooked sturgeon meat were summarized in Table 3. The relative numbers of observed modified peptides found were presented in Fig. 3. In general, various types of modifications were promoted at the primary structure level of actin when the sturgeon fillets were subject to heat treatment, involving introduction of carbonyl moieties to lysine, histidine and arginine residues, oxidation of the sulfur-containing residues, modification of aromatic residues, notably tryptophan and tyrosine, and formation of advanced glycation endproducts (AGEs). Moreover, some differences in type and quantity of modifications were detected as roasted and fried ones exhibited a greater content and diversity of modifications, 
Table 3 Heating and/or reactive oxygen species-induced modifications in sturgeon fillets after cooking (boiling, steaming, microwaving, roasting and frying $)^{a}$

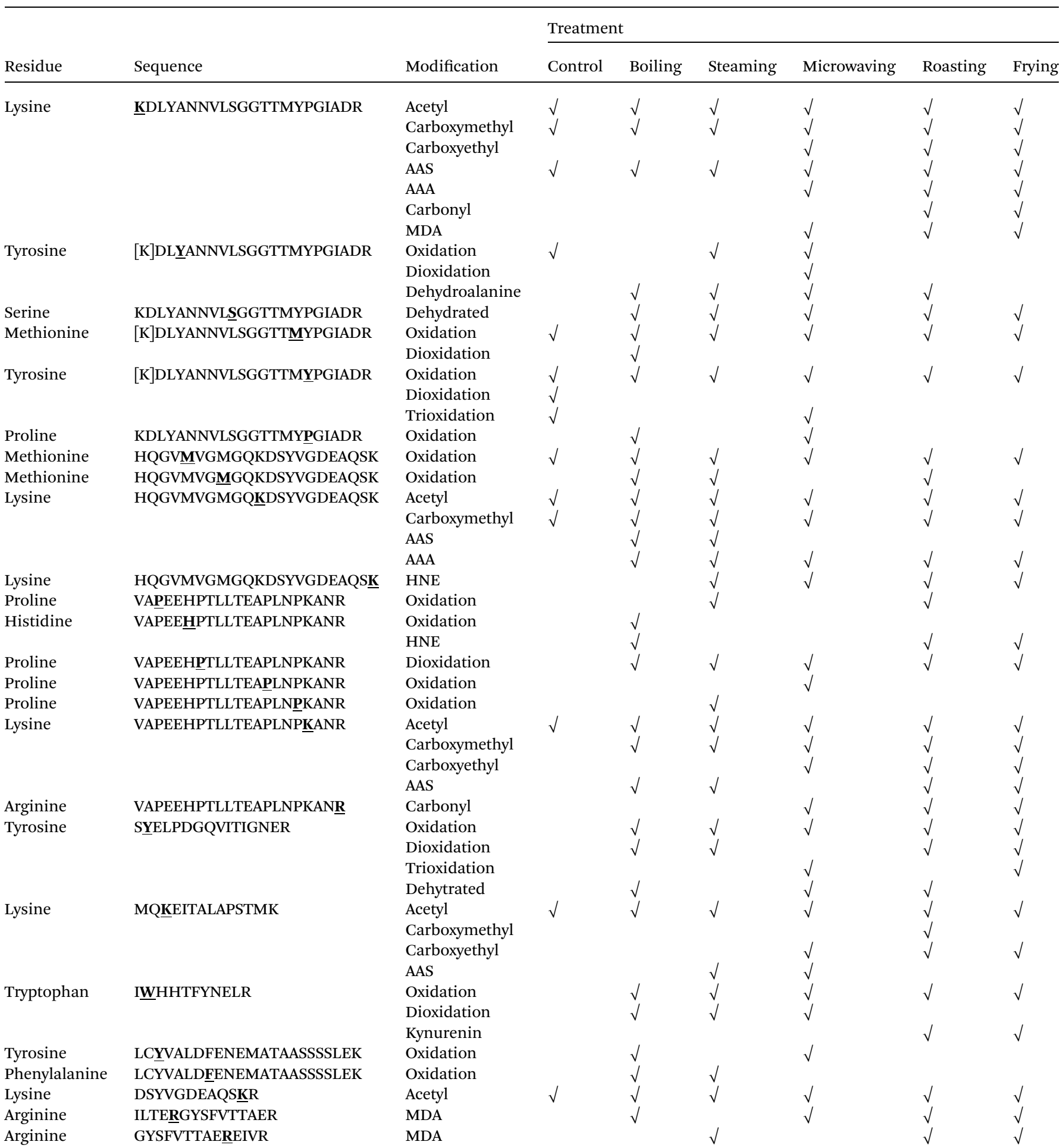

${ }^{a} \sqrt{ }$ means that the modification was found, and blank means that a modification was not found. Residues contained within square brackets represent extensions of the peptide that were also observed.

but relatively low levels of oxidative modification were detected in boiled and steamed samples. However, we also found oxidation of some residues in the control samples, indicating that some background level of oxidative modifications occurred in the raw samples.
Table 3 showed lysine residues were readily modified into compounds containing carbonyl groups and the major sites of such modifications in actin were within 4 tryptic peptides: KDLYANNVLSGGTTMYPGIADR, VAPEEHPTLLTEAPLNPKANR, MQKEITALAPSTMK and HQGVMVGMGQKDSYVGDEAQSK. $\alpha$ - 


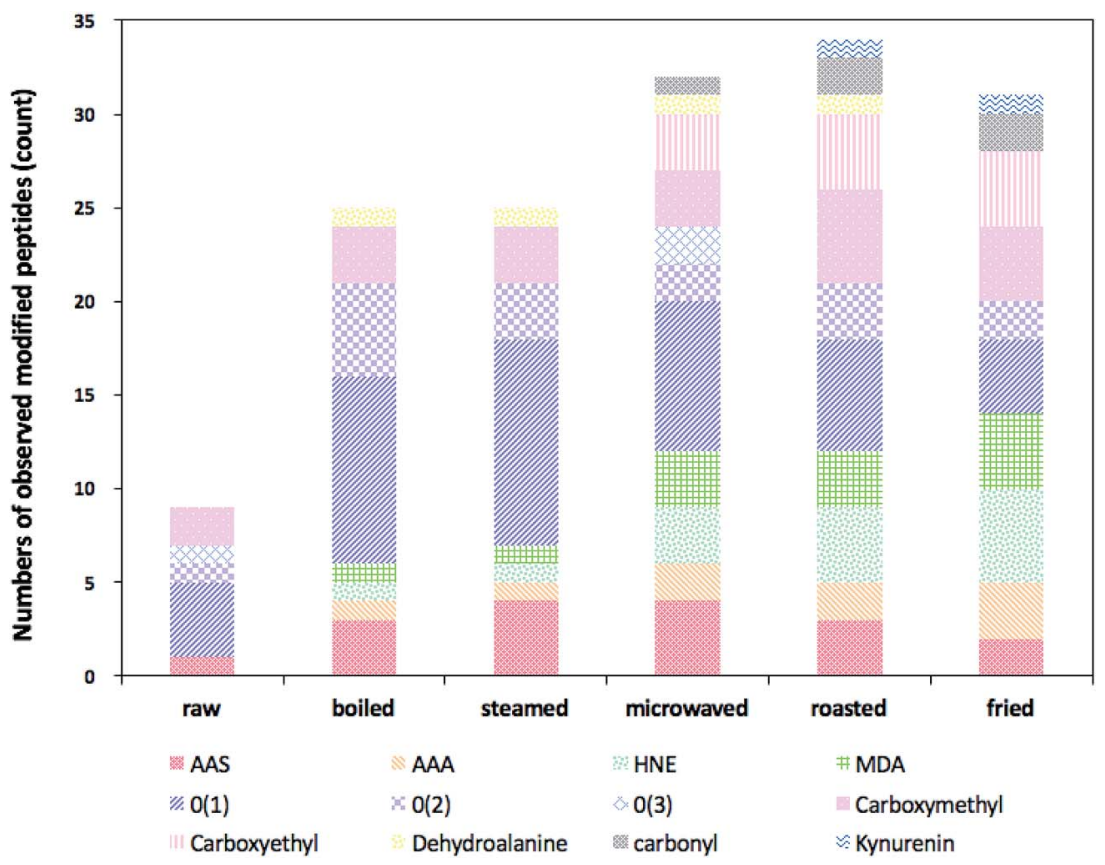

Fig. 3 Relative numbers of observed modified peptides of protein extracts from raw sturgeon fillets and those cooked with different cooking methods (AAS: $\alpha$-aminoadipic semialdehyde; AAA: $\alpha$-aminoadipic semialdehyde; HNE: 4-hydroxy-2-nonenal; MDA: malondialdehyde; O(1): oxidation; $O(2)$ : dioxidation; $O(3)$ : trioxidation).

Aminoadipic acid semialdehyde (AAS) is one of the major carbonyl derivatives of lysine which is formed in processed meat as a result of the oxidative deamination of the side chains from lysine residues. In the present study, AAS was detected in all the samples with cooked ones carrying a larger proportion than the raw (Fig. 3). According to Estévez, ${ }^{29}$ AAS, undergoes a further oxidative degradation under intense oxidative conditions (i.e., induced by hydroxyl radical-generating systems) lead to formation of a stable end-product: $\alpha$-aminoadipic acid (AAA), explaining why in our study, no AAA was found in the raw meat, while higher levels of AAA were found in roasted and fried samples compared to other types of cooked samples.

Lysine can also be modified by covalent reactions with oxidized sugars, often referred to as AGE. ${ }^{35} N \varepsilon$-Carboxymethyllysine (CML) and $N \varepsilon$-carboxyethyllysine (CEL), are 2 common AGEs. Carboxymethylation of lysine residues was detected in all the samples and carboxyethylation of lysine was identified only in cooked samples (Fig. 3). Similarly, higher levels of these two modifications were found in roasted and fried ones, which means more AGEs were produced during roasting and frying.

In addition, we identified and tracked a variety of other common protein redox modifications. These were summarized in Table 3 and Fig. 3. Among all the residues, aromatic amino acids are the major focuses of our study since they have been reported to be particularly sensitive to the oxidative processes associated with ROS attack. Tryptophan, phenylalanine and tyrosine are three aromatic amino acids of which the former two are essential for human. In the present study, we identified a range of different oxidative modifications in aromatic amino acids residues. Oxidation of tryptophan occurred in all cooked samples and di-oxidation products existed in boiled, steamed and microwaved ones. Kynurenine, a toxic tryptophan oxidation product was only found in samples under roasting and frying. In addition, oxidation, di-oxidation and even tri-oxidation of tyrosine were also observed in cooked samples and oxidized phenylalanine was characterized in boiled and steamed samples. Proline, in our experiment, was vulnerable to oxidation as well, for different types of oxidized proline residues were detected in several cooked samples.

On the whole, direct oxidation by incorporating a different number of oxygen atoms into amino acids residues extensively occurred in all the samples, and such kind of oxidation was more prevailing in samples under mild cooking condition like boiling and steaming, but less in microwaved, roasted and fried ones. Probably, those initially oxidized amino acids in boiled and steamed samples were reactive and tended to undergo further reaction under Hasher conditions like roasting and frying.

Apart from above redox modifications, 4-HNE-modified and MDA-modified peptides were identified in cooked fillets (Fig. 3), with levels of these two modifications elevated in samples cooked by microwaving, roasting and frying, which delivered a clear clue of the interconnection between lipid oxidation and protein oxidation. Both 4-HNE and MDA are two of the most abundant secondary products (toxic aldehydes) generated through lipid peroxidation when the lipids are faced with oxidative stress like high temperature. However, they are highly reactive and electrophilic, capable of modifying nucleophilic residues within proteins, like cysteine, histidine, lysine and arginine $^{36}$ 4-HNE and MDA which contain carbonyl groups (aldehydes or ketones) and $\alpha, \beta$-unsaturated moieties can form 
a variety of adducts with proteins. Although lipid-protein oxidation has already been explored in many studies, such interactions have been poorly studied in food systems.

In medical research, aforementioned oxidative modifications of protein including protein lipoxidation have been widely studied and associated with aging and some pathological conditions as Alzheimer's disease. Whether the dietary oxidizing proteins will be a source of disease still remain to be elucidated. Nevertheless, we can't deny that pinpointing protein modifications is significant for muscle foods since their nutritional value would be compromised. On one hand, inferior digestibility would be caused by modifications of amino acids residues both directly and indirectly. Many modified residues are the right sites for hydrolysis by digestive enzymes, and protein aggregation induced by cross-links between modified residues would bury the hydrolysis sites, which wards them off further breaking down upon the digestive enzymes. On the other, bioavailability of amino acid residues will be impaired and some essential amino acids will be destroyed, even worse, toxic compounds will form. Multiple unnaturally oxidized amino acids were reported to be potentially toxic. Wang et al. ${ }^{37}$ found AAA was strongly associated with the risk of developing diabetes. HNE-bound protein was associated with many diseases. ${ }^{38}$ The neurotoxicity of kynurenines - oxidation products of tryptophan - and their relevance in the gastrointestinal diseases was also well covered..$^{39}$ The accumulation of these potentially toxic compounds during cooking and their absorption upon food digestion could probably have an implication on human health.

\section{Conclusions}

Cooking resulted in a significant increase of carbonyls and Schiff bases as well as a notable loss of free thiols in fish fillets compared to the raw material. The extent of oxidation was affected by five different cooking methods among which roasting and frying made the fish protein more susceptible to oxidation which was evidenced by much higher content of carbonyls and Schiff bases and lower levels of free thiols. Moreover, proteomic study further explored the mechanism of cooking-induced protein oxidation. Roasting and frying gave rise to a higher level and a greater diversity of modifications in fish fillets, but a relatively low level in boiled and steamed samples. Side-chain modifications of amino acid residues including oxidation of phenylalanine, tryptophan, tyrosine and extensive oxidation of lysine leading to the formation of AAS, AAA. CML and CEL were characterized in the cooked samples. Indirect protein oxidation caused by interacting with lipidderived aldehydes like MDA and HNE was also observed and much more pronounced in roasted and fried samples which also explained the relatively low TBARS value detected in those samples, providing a footprint of the interaction between lipid oxidation and protein oxidation.

\section{Conflict of interest}

The authors declare that they have no conflicts of interest.

\section{Acknowledgements}

This work was supported by the National Science-technology Support Plan project (2014BAD04B01).

\section{References}

1 Y. Xiong and E. A. Foegeding, J. Food Sci., 2015, 80, iii.

2 R. Domínguez, M. Gómez, S. Fonseca and J. M. Lorenzo, Meat Sci., 2014, 97, 223-230.

3 S. Alsaghir, K. Thurner, K. Wagner, G. Frisch, W. Luf, E. Razzazifazeli, et al., J. Agric. Food Chem., 2004, 52, 52905296.

4 C. Diaconescu, G. Fantaneru, L. Urdes, V. Livia, B. Vasile and D. Stefan, Rom. Biotechnol. Lett., 2013, 18, 8279-8283.

5 M. Estévez and C. Luna, Crit. Rev. Food Sci. Nutr., 2016, DOI: 10.1080/10408398.2016.1165182.

6 V. H. Kjærsgård, M. R. Nørrelykke, C. P. Baron and F. Jessen, J. Agric. Food Chem., 2006, 54, 9437-9446.

7 S. Traore, L. Aubry, P. Gatellier, W. Przybylski, D. Jaworska, et al., Meat Sci., 2012, 91, 14-21.

8 S. H. Hernández-López, J. G. Rodríguez-Carpena, C. LemusFlores, J. Galindo-García and M. Estévez, Antioxidant protection of proteins and lipids in processed pork loin chops through feed supplementation with avocado, J. Food Sci. Technol., 2016, 53, 1-9.

9 M. Roldan, T. Antequera, M. Armenteros and J. Ruiz, Food Chem., 2014, 149, 129-136.

10 P. Gatellier, V. Sante-Lhoutellier, S. Portanguen and A. Kondjoyan, Meat Sci., 2009, 83, 651-656.

11 P. Gatellier, A. Kondjoyan, S. Portanguen and V. SanteLhoutellier, Meat Sci., 2010, 85, 645-650.

12 P. Gatellier, A. Kondjoyan, S. Portanguen, E. Grève, K. Yoon and V. Santé-Lhoutellier, Food Chem., 2009, 114, 1074-1078.

13 X. Sun, J. Tang, J. Wang, B. A. Rasco, K. Lai and Y. Huang, Food Chem., 2015, 172, 802-807.

14 J. M. Dyer, S. Clerens, A. Grosvenor, A. Thomas, C. Callaghan, S. Deb-Choudhury, et al., J. Dairy Sci., 2016, 99, 3295-3304.

15 J. M. Dyer, S. Clerens, A. Thomas, C. Callaghan, S. DebChoudhury and S. Haines, Int. Dairy J., 2016, 66, 56-60.

16 S. Deb-Choudhury, S. Haines, D. Harland, S. Clerens, C. van Koten and J. Dyer, J. Agric. Food Chem., 2014, 62, 8187-8196.

17 T. Y. Yu, J. D. Morton, S. Clerens and J. M. Dyer, J. Agric. Food Chem., 2015, 63, 9112-9123.

18 T. Y. Yu, J. D. Morton, S. Clerens and J. M. Dyer, Meat Sci., 2016, 119, 80-88.

19 M. Nikoo and M. R. Ghomi, Rev. Nutr., 2013, 26, 67-74.

20 X. F. Lan, G. F. Ruan, Z. H. Fan, N. N. Li and S. Wang, Food Sci., 2015, 37, 16-22.

21 R. Ganhão, M. Esteévez and D. Morcuende, Food Chem., 2011, 126, 772-778.

22 Y. Mercier, P. H. Gatellier, M. Viau, H. Remignon and M. Renerre, Meat Sci., 1998, 48, 301-317.

23 M. Morzel, P. H. Gatellier, T. Sayd, M. Renerre and E. Laville, Meat Sci., 2006, 73, 536-543. 
24 D. M. Miller, J. V. Gomezbasauri, V. L. Smith, J. Kanner and D. Miller, J. Food Sci., 1994, 59, 747-750.

25 C. Guyon, A. Meynier and M. de Lamballerie, Trends Food Sci. Technol., 2016, 50, 131-143.

26 M. Utrera, D. Morcuende and M. Estévez, LWT-Food Sci. Technol., 2014, 56, 62-68.

27 K. Viljanen, R. Kivikari and M. Heinonen, J. Agric. Food Chem., 2004, 52, 1104-1111.

28 Y. Xiong, Protein oxidation and implications for muscle foods quality, in Antioxidants in muscle foods, ed. E. A. Decker, C. Faustman and C. J. Lopez-Bote, Wiley, New York, 2000, pp. 85-111.

29 M. Estévez, Meat Sci., 2011, 89, 259-279.

30 W. Zhang, S. Xiao and D. U. Ahn, Crit. Rev. Food Sci. Nutr., 2013, 53, 1191-1201.

31 M. E. Bailey, S. Y. Shin-Lee, H. P. Dupuy, A. J. St. Angelo and J. R. Vercellotti, Inhibition of warmed-over flavour by Maillard reaction products, in Warmed-over flavour of meat, ed. A. J. St. Angelo and M. E. Bailey, Academic Press, INC., Berlin, 1987, pp. 141-164.
32 S. Wen, G. Zhou, L. Li, X. Xu, X. Yu, Y. Bai, et al., J. Agric. Food Chem., 2015, 63, 250-261.

33 K. Kajak-Siemaszko, L. Aubry, F. Peyrin, M. L. Bax, P. Gatellier, T. Astruc, et al., Food Res. Int., 2011, 44, 31603166.

34 F. Huang, M. Huang, X. Xu and G. Zhou, J. Sci. Food Agric., 2011, 91, 443-448.

35 G. Vistoli, M. D. De, A. Cipak, N. Zarkovic, M. Carini and G. Aldini, Free Radical Res., 2013, 47, 3-27.

36 G. Aldini, M. R. Domingues, C. M. Spickett, P. Domingues, A. Altomare and F. J. Sanchez-Gomez, Redox Biol., 2015, 5, 253-266.

37 T. J. Wang, D. Ngo, N. Psychogios, A. Dejam, M. G. Larson, R. S. Vasan, et al., J. Clin. Invest., 2013, 123, 4309-4317.

38 G. Barrera, S. Pizzimenti, E. S. Ciamporcero, M. Daga, C. Ullio and A. Arcaro, Antioxid. Redox Signaling, 2015, 22, 1681.

39 D. Keszthelyi, F. J. Troost, D. M. Jonkers, E. L. van Donkelaar, J. Dekker, W. A. Buurman, et al., Am. J. Clin. Nutr., 2012, 95, 603-608. 